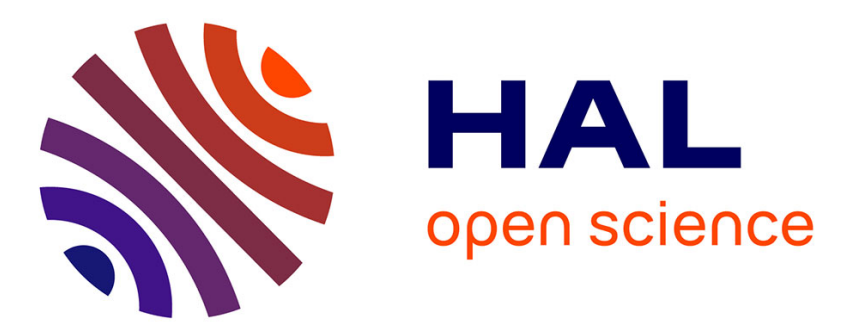

\title{
Fin de transition? Les élections législatives du 13 mai 2001 en Italie
}

\author{
Carlo Baccetti, Emmanuel Négrier
}

\section{To cite this version:}

Carlo Baccetti, Emmanuel Négrier. Fin de transition? Les élections législatives du 13 mai 2001 en Italie. Pôle Sud - Revue de science politique de l'Europe méridionale, 2001, La Commission Européenne en politique(s), 15, pp.119-131. 10.3406/pole.2001.1128 . hal-02551200

\author{
HAL Id: hal-02551200 \\ https://hal.science/hal-02551200
}

Submitted on 28 Apr 2020

HAL is a multi-disciplinary open access archive for the deposit and dissemination of scientific research documents, whether they are published or not. The documents may come from teaching and research institutions in France or abroad, or from public or private research centers.
L'archive ouverte pluridisciplinaire HAL, est destinée au dépôt et à la diffusion de documents scientifiques de niveau recherche, publiés ou non, émanant des établissements d'enseignement et de recherche français ou étrangers, des laboratoires publics ou privés.

\section{(ㅇ)(1) $\$$}

Distributed under a Creative Commons Attribution - NonCommercial - NoDerivatives 44.0 


\title{
Fin de transition ? Les élections législatives du 13 mai 2001 en Italie
} Mr Carlo Baccetti, Mr Emmanuel Négrier

\begin{abstract}
Italian Parliament elections of may 2001 have been the last step of a long political transition. Won by Berlusconi, the richest political man and Italian private entrepreneur, leading a centre-right coalition, these election have led to a strong political majority. This article deals with de social and political components of both coalitions. It then explains the reasons of the centerright coalition success, as well as the failure of center-left coalitions and that of the third way attempt, represented by two little parties which are now marginalized.
\end{abstract}

\section{Résumé}

Les élections législatives du 13 mai 2001 a mis un terme à la longue transition politique italienne. Elles ont été remportées par la coalition de centre-droit, dénommée Casa délie Liberta (Maison des Libertés), dirigée par Silvio Berlusconi, richissime entrepreneur- homme politique, qui préside désormais un gouvernement issu d'une solide majorité parlementaire. Cet article présente la composition sociale et politique de chacun des deux coalitions, explique les raisons du succès du centre-droit, l'échec de la gauche et de la troisième voie, incarnée par deux petits partis aujourd'hui marginalisés.

\section{Citer ce document / Cite this document :}

Baccetti Carlo, Négrier Emmanuel. Fin de transition ? Les élections législatives du 13 mai 2001 en Italie. In: Pôle Sud, n¹5, 2001. La Commission Européenne en politique(s) pp. 119-131;

doi : https://doi.org/10.3406/pole.2001.1128

https://www.persee.fr/doc/pole_1262-1676_2001_num_15_1_1128

Fichier pdf généré le 23/04/2018 


\section{Fin de transition? \\ Les élections législatives \\ du 13 mai 2001 en Italie}

Carlo Baccetti

Université de Florence

(Traduit de l'italien par Emmanuel Négrier)

\section{Résumé/Abstract}

Les élections législatives du 13 mai 2001 a mis un terme à la longue transition politique italienne. Elles ont été remportées par la coalition de centre-droit, dénommée Casa delle Libertà (Maison des Libertés), dirigée par Silvio Berlusconi, richissime entrepreneurhomme politique, qui préside désormais un gouvernement issu d'une solide majorité parlementaire.

Cet article présente la composition sociale et politique de chacun des deux coalitions, explique les raisons du succès du centre-droit, l'échec de la gauche et de la troisième voie, incarnée par deux petits partis aujourd'hui marginalisés.

Italian Parliament elections of may 2001 have been the last step of a long political transition. Won by Berlusconi, the richest political man and Italian private entrepreneur, leading a centre-right coalition, these election have led to a strong political majority. This article deals with de social and political components of both coalitions. It then explains the reasons of the center-right coalition success, as well as the failure of center-left coalitions and that of the third way attempt, represented by two little parties which are now marginalized.

\section{$\approx$ \\ Mots-Clés/Keywords}

Élections, Italie, modes de scrutin, partis politiques, sociologie électorale, Toscane Elections, electoral rules, electoral sociology, Italy, political parties, Tuscany 
Le résultat des législatives du 13 mai 2001 a mis un terme à la longue transition politique italienne. On sait qu'elles ont été remportées par la coalition de centre-droit, dénommée Casa delle Libertà (Maison des Libertés), dirigée par Silvio Berlusconi, richissime entrepreneur-homme politique, qui préside désormais un gouvernement issu d'une solide majorité parlementaire. Berlusconi jouit d'une large popularité et d'un soutien certain dans le pays, et il peut en outre compter sur l'influence de son parti "personnel": Forza Italia, qu'il a fondé, financé et contrôlé absolument. Forza Italia a fait un bond en avant de $9 \%$, au grand dam de ses alliés, et est devenu de loin le premier parti italien. En somme, après une décennie de bouleversements politiques qui ont totalement modifié le système des partis (en introduisant en outre de nouvelles règles électorales à tous les niveaux institutionnels, du Parlement aux municipalités), en entrânnant un changement significatif de la classe politique, après quatre élections générales, la transition italienne a accompli sa révolution: le système politique semble désormais suffisamment stabilisé et la majorité issue des urnes le 13 mai paraît assez compacte pour durer toute une législature.
Avant de commenter les données les plus importantes, il convient de rappeler que cette victoire du centre-droit était largement annoncée, qu'il s'agisse des sondages, tous favorables à Berlusconi, ou bien des élections immédiatement antérieures: les européennes de 1999 ou les régionales de 2000. Ces dernières, considérées comme une dernière répétition avant les législatives, ont été remportées très nettement par le centre-droit, qui était de ce fait majoritaire dans le pays (Baccetti, Caciagli, 2000). Les régionales ont également marqué une étape importante: le retour de la Lega Nord, le mouvement séparatiste dirigé par Umberto Bossi, dans l'alliance de centredroit, aux côtés de Forza Italia, des post-fascistes d'Alleanza Nazionale et des centristes ex-démocrates-chrétiens du CCD-CDU. Bossi avait pris acte du fait que son mouvement perdait de plus en plus de terrain au profit de Forza Italia, et avait accepté ce "retour au bercail", dans une position clairement subalterne de Berlusconi. Après la rupture provoquée par Bossi fin $1994^{1}$ - la vraie trahison selon Berlusconi -, l'alliance s'était donc recomposée, sur la base d'une motivation pragmatique: se présenter unis était la seule manière de ne pas risquer de perdre à nouveau les élections. Étant donné le méca- 
nisme électoral en vigueur, en fait, la capacité de signer des accords électoraux couvrant toutes les circonscriptions représente indubitablement l'un des facteurs qui détermine le plus sûrement le résultat électoral et décide de la stabilité ou de l'instabilité du gouvernement.

Passant, avec désinvolture, sur leurs graves insultes mutuelles et leurs profondes divergences et rivalités politiques, les leaders des quatre partis de la Casa delle Libertà avaient donc reproposé aux électeurs l'ancien cartel politique vainqueur en 1994. Et les électeurs, chose plus importante, en avaient en majorité montré de la satisfaction. Après un tel succès électoral, le plus dur était fait, non seulement parce que la victoire avait été nette $(50,6 \%$ des voix dans les 15 régions à statut ordinaire où l'on votait), mais aussi parce que la défaite contraignit Massimo D'Alema, le chef du gouvernement de l' Ulivo (l'Olivier, coalition de centre-gauche), à la démission. Celui-ci avait imprudemment déclaré que ces élections étaient de fait un pronuciamento pour ou contre son gouvernement. L'Ulivo avait subi le contrecoup négatif de cette défaite, et s'était retrouvé, à moins d'un an des échéances, sans leader à opposer à Berlusconi. Pour consolider et "tenir au chaud" son influence, Berlusconi avait occupé sans relâche la scène médiatique, facilité en cela par ses moyens financiers et audio-visuels.

La Casa delle Libertà a donc pu tirer pleinement avantage du mécanisme uninominal majoritaire: à la Chambre, avec $45,4 \%$ des voix, elle a obtenu $59,4 \%$ des 475 sièges du quota majoritaire (soit 282), auxquels on doit ajouter les 82 sièges sur 155 obtenus au titre du quota proportionnel. Au total, la majorité de centre-droit s'y établit à 368 sièges sur 630 , soit $58,4 \%$. Au Sénat, elle détient, avec $42,5 \%$ des voix, $55,9 \%$ des sièges (176 sur 315).
Pour sa part, la coalition de centre-gauche n'est en revanche pas parvenue à renouveler l'alliance électorale avec Rifondazione Comunista, le parti néo-communiste dirigé par Fausto Bertinotti. L'Ulivo s'est ainsi présenté aux élections avec quatre composantes, dont deux étaient des regroupements de partis: les Démocrates de Gauche (DS), le Parti des Communistes Italiens (lui-même issu d'une scission de Rifonsazione Comunista en 1998), le Girasole (le Tournesol, une alliance électorale composée des Verts et des socialistes italiens), et la Margherita, une coalition regroupant en une seule liste quatre partis centristes: le PPI, Udeur, Rinnovamento Italiano et I Democrati (les démocrates). L'Ulivo a perdu, par rapport à 1996 (où l'alliance existait avec Rifondazione Comunista), un peu plus de 600000 voix et $1,6 \%$ dans les collèges uninominaux de la Chambre, où Rifondazione avait renoncé "unilatéralement" de présenter des candidats. Dans les collèges sénatoriaux, en revanche, celui-ci s'était présenté en concurrent à gauche de l'Ulivo: si l'on additionnait les voix au Sénat, on constaterait que le recul est encore plus limité par rapport à 1996: 0,7 \% de moins, alors qu'en termes absolus, l'Ulivo et Rifondazione ont recueilli ensemble environ 400000 voix de plus que cinq ans auparavant, lorsqu'ils s'étaient présentés unis.

Donc, après deux élections (1994 et 1996) desquelles avaient découlé des majorités étriquées et des gouvernements faibles et instables, la loi électorale mixte, si contestée, a bien fonctionné, produisant une majorité solide au gouvernement.

Les chiffres du 13 mai nous indiquent cependant que la victoire de la Casa delle Libertà a été plus nette sur le plan politique qu'en termes électoraux. Comme on peut le voir dans les tableaux 1 et 2 , dans la partie majoritaire de la compétition ${ }^{2}$, l'écart entre les 
deux pôles est réduit: à la Chambre, les candidats de centre-droit ont devancé leurs adversaires de seulement 600000 voix, soit $1,7 \%$. Au Sénat, l'écart est certes plus grand $(1,1$ millions de voix, soit $3,3 \%)$, mais la gauche y est majoritaire si l'on cumule les voix de l'Ulivo et celles de Rifondazione 3 . Par rapport aux précédentes élections de 1996, la Casa delle Libertà est en outre restée bien en deçà du cumul des voix du Pôle des Libertés et de la Lega Nord, lorsqu'ils s'étaient présentés séparément: 600000 voix de moins à la Chambre (2\%), 1,7 millions au Sénat (7\%). Quant au vote proportionnel (tableau $n^{\circ} 3$ ), il montre que tous les partis composant la Casa delle Libertà ont largement perdu, à l'exception notable de Forza Italia. Dans l'ensemble, la coalition a chuté de $2,6 \%$ et 1,1 millions de voix par rapport au cumul Pôle-Lega de 1996.

\section{La zone rouge: la Toscane}

Il n'est pas sans intérêt, dans cette discussion sur le vote de gauche, de regarder sa distribution territoriale, et plus particulièrement dans la zone rouge (Emilie-Romagne, Toscane, Ombrie et Marches), historiquement connue pour l'enracinement territorial d'une subculture politique socialiste, puis communiste qui a toujours assuré à la gauche, puis au centre-gauche (depuis 1994) un niveau élevé d'influence électorale et la conquête de tous les sièges parlementaires, ou peu s'en faut. En fait, les liens d'appartenance subculturelle à la gauche se sont depuis longtemps relâchés, sinon quasi-totalement défaits. Des indices clairs de cette tendance - le recul du militantisme et de la participation politique dans les partis de gauche - étaient apparus dès avant 1989, lorsqu'explosa la crise et la transformation organisationnelle du PCI. Toutefois, en termes de comportement électoral, les liens avec la tradition territoriale avaient continué de jouer dans les années 1990, et les partis néo et post-communistes avaient ensemble confirmé leur hégémonie. Avec les élections du 13 mai, des signaux inquiétants pour la gauche sont apparus. Les racines culturelles, sociales et territoriales qui avaient garanti la continuité du même comportement électoral semblent s'être usés, en bien des cas. Nous pouvons observer que les choses continuent d'aller assez bien, de fait, dans la compétition majoritaire où, pour les quatre régions, ' 'Ulivo conquiert la presque totalité des sièges en jeu ( 74 députés sur 80,36 sénateurs sur 40 ) et conserve, en compagnie de Rifondazione Comunista, la majorité absolue des voix, tout en perdant $2 \%$ par rapport à 1996 , avec $56 \%$ des voix). Le centre-droit, quant à lui, demeure distancé avec $40 \%$ environ dans chaque région. Toutefois, la nouveauté apparaît au niveau du vote proportionnel : la suprématie de l' Ulivo est de plus en plus assurée par sa composante centriste (la Margherita), et de moins en moins par les partis de gauche. La première y passe de 11 à $15 \%$, tandis que les derniers chutent de $8 \%$ (à $36 \%$ ), soit 600000 voix de moins qu'en $1996^{4}$. Des régions certes encore rouges (ou mieux : roses), mais tendanciellement toujours moins rouges et plus "ulivistes".

Dans cette baisse générale des partis issus du PCI dans la zone rouge, la région qui perd le moins est la Toscane. Si bien qu'elle a surpassé l'Émilie-Romagne et est devenue, maigre consolation, la région la plus rouge d'Italie.

Ceci s'est surtout produit grâce aux DS, qui ont enregistré une baisse moins forte en Toscane $(30,9 \%$ en 2001 contre $34,7 \%$ en 1996, alors que le même rapport est, en Émilie-Romagne, de 28,8\%/35,6\%). Certes, un recul de 3,8\% (soit 115000 électeurs de moins) n'est pas négligeable, mais il 
est inférieur de moitié à ce que l'on observe dans les trois autres régions.

La perte d'influence de Rifondazione Comunista est plus forte. Son implantation très forte en Toscane (12,5\% en 1996) a été ramenée à $6,9 \%$. Il faut sans doute leur ajouter les 2,3\% des Communistes Italiens (CI) d'Armando Cossutta. Au total, les néo-communistes ( $\mathrm{RC}$ et $\mathrm{CI}$ ) perdent $3,2 \%$, soit 317000 électeurs.

DS, RC et CI ont recueilli en Toscane 1 millions de voix, soit $40,1 \%$ (contre $35,9 \%$ en Emilie-Romagne), dépassant de peu le score de 39,3\% obtenu, lors des difficiles élections de 1992, par le PDS et Rifondazione - alors qu'ils avaient atteint $43,8 \%$ en 1994 et $47,2 \%$ en 1996 . Ceci s'est accompagné d'un niveau de participation électorale inférieur aux précédentes législatives $(86,5 \%$ contre $88,4 \%$ ), même s'il avait largement remonté par rapport aux régionales de 2000.

En somme, s'il est vrai qu'en Toscane "l'implantation politique et électorale de la gauche a globalement mieux résisté qu'ailleurs", l'évolution du vote de gauche, même dans la région qui constitue son bastion, montre que le lien entre parti et société n'est plus le même, et que son influence électorale est devenue pour le moins instable. L'analyse des votes à l'échelle sub-régionale laisse entendre que ces partis ne sont pas parvenus à rénover leur implantation territoriale, à retisser des liens organisationnels avec les nouvelles figures sociales et professionnelles qui étaient venus, dans les années 1990 , se substituer aux traditionnels groupes sociaux de la région. Pour ne mentionner qu'un seul fait, peut-être le plus significatif: les pertes les plus graves des DS se situent presque toutes dans les zones qui sont réellement le "cœur de la subculture rouge ${ }^{6}$ ". Rifondazione, ellemême, a enregistré ses plus importants reculs là où elle avait la plus grande influence.
La Toscane délivre donc un message clair aux formations de la gauche "historique": les changements sociaux et le processus de modernisation ont définitivement érodé une grande part des liens d'appartenance subculturelle; même si cela ne signifie pas que la Toscane ait "changé de peau", ou soit devenue de centre-droit. Au contraire, le vote du 13 mai a confirmé encore que le message de Berlusconi ne parvient pas à passer: la culture politique démocratique, l'esprit civique représentent un enracinement solide qui survit à l'épuisement des identifications partisanes. C'est le rapport aux partis qui s'est en revanche affaibli. Il est devenu plus critique, et même conflictuel : les électeurs de gauche sont prêts à se réfugier dans l'abstention ou à s'orienter vers une offre différente, en restant pourtant dans le même espace politique de centre-gauche, si la proposition de leurs partis de référence ne les convainc pas assez.

\section{Les deux électorats}

Derrière les chiffres sont les électeurs. Les informations tirées de plusieurs recherches nous livrent plusieurs enseignements sur les caractéristiques des électeurs de la Casa delle Libertà et de l'Ulivo. En particulier, les premières leçons d'une importante recherche menée par le groupe ITANES (de l'Institut Cattaneo de Bologne ${ }^{7}$ ), basée sur des interviews post-électorales d'un échantillon de 3200 personnes, qui identifie de profondes différences entre deux électorats, analysés selon trois dimensions: les caractéristiques démographiques, les conditions socio-économiques et l'exposition aux médias. L'élément sans doute le plus important et surprenant, qui démentit plusieurs études conduites jusqu'alors en Italie, est que les jeunes (jusqu'à 24 ans - notamment les étudiants, qui sont la majeure partie de cette tranche d'âge -) n'ont 
pas voté pour la Casa delle Libertà mais nettement pour l'Ulivo. Plus généralement, on peut dire que l'âge est un bon indicateur pour expliquer le choix de vote: les trentenaires votent nettement à droite, les quarantenaires se partagent équitablement entre les deux pôles et les plus âgés votent à droite.

Si l'âge influence grandement les choix, ce n'est pas le cas du genre, surtout pour les hommes, qui se répartissent équitablement entre droite et gauche. Les femmes ont une légère préférence pour la gauche ce qui, pour les chercheurs, manifeste le croisement de deux tendances opposées: les femmes au foyer votent à droite, les femmes actives à gauche ${ }^{8}$.

Mais les deux variables largement plus significatives pour expliquer le vote sont la catégorie professionnelle et le niveau d'information politique et d'exposition aux médias. L'étude ne laisse aucun doute: I' Ulivo dispose d'une chasse gardée chez les salariés du secteur public, enseignants et employés en premier lieu; les salariés du secteur privé sont partagés, tandis que les travailleurs indépendants sont nettement favorables à Silvio Berlusconi "au niveau élevé (professions libérales et entrepreneurs) comme aux niveaux inférieurs (commerçants et artisans $\left.{ }^{9}\right)^{\prime}$.

C'est la fracture politique qui semble la plus importante, notamment parce qu'elle est relativement nouvelle, dans la société italienne : d'un côté les travailleurs "garantis" du secteur public qui votent à gauche; de l'autre les travailleurs autonomes qui "font quotidiennement l'expérience des lois du marché" et se sentent représentés par un homme politiqueentrepreneur à succès comme Berlusconi. $58 \%$ des salariés du public ont choisi l'Ulivo, contre $31 \%$ pour la Casa delle Libertà. Au contraire, $56 \%$ du vaste groupe des indépendants a choisi cette dernière contre $34 \%$ l'Ulivo. Tandis que la distinction traditionnelle - les ouvriers votant à gauche et les employés à droite - a perdu son sens, les ouvriers de répartissent désormais également entre droite et gauche ${ }^{10}$.

Pour ce qui concerne enfin le degré d'information politique des citoyens, la distinction entre les deux fronts demeure nette: les plus informés et les plus instruits ont voté majoritairement pour l' Ulivo (les bacheliers et diplômés du supérieur ont respectivement voté avec des écarts pour la gauche de 7 et $9 \%$ ); cependant que "les électeurs qui ne disposent que de diplômes du secondaire ou de l'élémentaire, ont un bas niveau d'information politique, qui ne lisent pas les journaux et regardent beaucoup la télévision, votent majoritairement à droite ${ }^{11}$ ".

\section{Pourquoi la Casa delle Libertà a gagné ?}

On peut donc en déduire que la Casa delle Libertà a basé son succès sur le soutien de deux secteurs électoraux socialement, économiquement et culturellement très différents entre eux. D'un côté des catégories "marginales": femmes au foyer, retraités, habitant souvent de petits villes, peu instruits, etc. De l'autre, des groupes sociaux économiquement et socialement centraux (entrepreneurs, professions libérales et commerçants, etc.). Il est assez évident qu'un électorat aussi différencié ait voté pour la Casa delle Libertà sur la base de motivations, d'attentes et d'intérêts divers, voire contradictoires. Du reste, ces différences se reflètent dans les contrastes entre les bases électorales des quatre partis qui composent la coalition.

Il ne fait pas de doute que la "synthèse" politico-électorale de la Casa delle Libertà a été rendue possible en vertu de la capacité d'attraction exercée par la personne de Berlusconi. Tous reconnaissent que c'est lui qui a gagné, plus que le centre-droit. Il a commencé 
à construire sa revanche au travers d'une très longue campagne électorale, initiée dès la chute du gouvernement Prodi, en 1998. La campagne a cru en intensité, conduite avec le soutien évident de ses trois réseaux télévisuels et de ressources financières massives que seul Berlusconi - qui est non seulement l'homme politique mais aussi l'entrepreneur le plus riche d'Italie - pouvait se permettre. Depuis l'utilisation d'un bateau de croisière pour sa tournée électorale des régionales de 2000 à l'affichage, un an avant les législatives, dans toutes les villes importantes, de gigantesques publicités au haut desquelles surgissait le sourire de Berlusconi, opportunément arrangé esthétiquement, tout cela rassurait les italiens qui, avant qu'il fût nommé président du Conseil, étaient persuadés qu'ils allaient payer moins d'impôts, que les jeunes et les chômeurs allaient trouver du travail, que les retraites seraient augmentées, que les villes seraient plus sûres, etc. En vue des élections, Berlusconi a délibérément fait de l'ombre à tous les autres candidats et alliés, soutenant avec désinvolture que s'ils s'étaient montrés plus souvent, ils auraient plus risqué de faire perdre leur camp que de le faire progresser. Cela est allé jusqu'au "conseil" aux candidats de centre-droit de ne pas utiliser de photos d'eux-mêmes pour leurs campagnes, afin de mettre au maximum en évidence l'identification à la Casa delle Libertà à sa seule personne. En somme, Berlusconi a transformé les élections en défi personnel: lui, l'homme de succès, l'entrepreneur richissime, toujours gagnant contre la coalition réchauffée et confuse de centre-gauche, conduite par le "communiste" D'Alema, considéré comme le vrai chef de la coalition, y compris lorsque celui-ci a dû démissionner de la présidence du Conseil, et laisser diriger la coalition par le maire de Rome, Francesco Rutelli. En alternant contradictoirement, mais habilement, des promesses populistes et des messages néolibéraux, Berlusconi a convaincu la majorité des italiens à signer un contrat de cinq ans avec lui ${ }^{12}$, en se confiant la direction de 1'“entreprise Italie", qu'il saurait rendre prospère, comme il l'avait fait pour la sienne, créée à partir de rien ${ }^{13}$.

\section{Pourquoi l'Ulivo a perdu}

Si les raisons de la victoire du centre-droit se résument toutes, en substance, au travers de la personne de son leader, les raisons de la défaite de l'Ulivo sont variées.

Un regard rétrospectif sur l'expérience de cinq années de gouvernement pourrait suggérer que l'Ulivo a commencé à perdre les élections 2001 dès octobre 1998, à la chute du gouvernement Prodi, le seul des quatre gouvernements de la XIII législature à avoir reçu son investiture directement de l'électorat. La chute du premier gouvernement a été provoquée par Rifondazione Comunista, qui décida de lui retirer son indispensable soutien parlementaire. Le choix d'éliminer Prodi fut suivi par une scission interne qui l'affaiblit beaucoup; mais la sortie de scène de Prodi coûta aussi, dans le peuple de gauche et l'électorat progressiste en général, en espérances, suscitées par la naissance de l'Ulivo, de renouvellement de la politique.

Le leader du PDS (puis DS), Massimo D'Alema avait lui-même porté un coup dur à de telles aspirations, à l'intérieur même de l'Ulivo. Au risque d'affaiblir l'action du gouvernement, il avait cherché avec insistance l'affrontement institutionnel avec l'opposition et Berlusconi en particulier, en ouvrant les travaux d'une Commission bicamérale pour les réformes institutionnelles, dont il s'était fait nommer président, avec le soutien de l'opposition. Les travaux de la Commission se conclurent (été 1998) par un échec et 
ne servirent finalement qu'à accréditer encore l'image de vainqueur de Berlusconi.

Les deux gouvernements de D'Alema et de Giuliano Amato, qui se sont succédés après Prodi, étaient soutenus par des majorités distinctes de celle de l'Ulivo et ne disposaient ainsi pas d'une investiture électorale, ce que ne se privait pas de dénoncer Berlusconi, qui réclama sans cesse des élections anticipées. Avec le passage de Rifondazione dans l'opposition, l'Ulivo ne disposait plus d'une majorité parlementaire. Pour pouvoir composer son équipe, D'Alema avait donc accepté de dénaturer l'alliance de centre-gauche en l'élargissant à une nouvelle formation politique du centre (l'UDR, puis Udeur, dirigée par l'ancien président de la République Cossiga, puis par l'ex-démocrate-chrétien Clemente Mastella), formée en grande partie de parlementaires de centre-droit (essentiellement du CCD-CDU, plus quelques-uns de Forza Italia sinon... d'Alleanza Nazionale). Politiquement contraint par le soutien de parlementaires et de ministres de centredroit, affaibli par les scissions et conflits au sein des formation qui le soutenaient, ce gouvernement de centre-gauche, s'il ne démérita pas en termes d'action institutionnelle, a contribué à miner le capital de sympathie et de participation populaire qui animait l'Ulivo à l'origine.

En outre, la coalition de centre-gauche - et c'est sûrement une raison de sa défaite - s'est montrée divisée et indécise sur la personne de son leader. L'Ulivo a donné des signes de désaccords jusque pendant la campagne électorale et, surtout, n'a pas fait beaucoup pour dissiper le soupçon qu'elle considérait la défaite inéluctable. La figure de Rutelli a émergé tardivement comme leader reconnu de la coalition et n'a ainsi jamais eu la capacité de mettre Berlusconi en difficulté - lequel a même pu se permettre de se soustraire à un débat télévisé risqué, sans pour autant perdre d'influence.

\section{Il n'y a pas de troisième force. Les listes d'Antonio Di Pietro et de Sergio D'Antoni}

La difficulté de donner vie à une coalition et de ne pas laisser la moindre marge à des listes concurrentes sur le même espace politique est le signe le plus le plus évident de la crise politique que traversait l' Ulivo, dès avant les élections. Cela est non seulement vrai pour l'accord manqué avec Rifondazione Comunista, mais se vérifie aussi au travers de la présence de deux listes qui se proposaient ouvertement d'en finir avec la logique bipolaire entre centre-gauche et centre-droit, en introduisant un troisième pôle dans le système politique, capable de peser sur les choix et l'orientation des deux autres pôles. Il s'agit ici des listes présentées, l'une (liste Di Pietro, Italia dei Valori) par l'ex-magistrat Antonio Di Pietro ${ }^{14}$, ennemi juré de Berlusconi, qui avait fait de la lutte contre la corruption son principal slogan, et l'autre (Democrazia Europea) par Sergio D'Antoni, secrétaire général de la CISL, second syndicat italien, d'inspiration catholique. D'Antoni s'était démis de sa charge au moment où, à l'automne 2000 , il avait annoncé son "entrée en politique" et la naissance de "son" parti dont l'objectif était de refaire la "Démocratie Chrétienne du XXI" siècle". Il avait appelé à lui tous les nostalgiques de l'existence d'un parti modéré comme la Démocratie-Chrétienne de l'âge d'or, afin qu'il occupât l'espace politique central, en s'alliant un jour à la gauche et l'autre à la droite au gré de la conjoncture et des nécessités politiques.

Ni la liste Italia dei Valori, ni la Democrazia Europea n'ont eu le succès électoral 
espéré, et n'ont enrayé l'affirmation de la logique bipolaire. Au contraire, la bipolarisation n'avait jamais connu une telle reconnaissance, tant et si bien que les deux listes "troisième voie" ont subi une déconfiture: comme on peut le voir dans les tableaux, ils ont non seulement conquis aucun siège au scrutin majoritaire (seuls deux sénateurs DE à la proportionnelle), mais il n'ont même pas pu accéder à la répartition proportionnelle des sièges de député. Leurs scores sont restés au-dessous des $4 \%$ requis à l'échelle nationale ${ }^{15}$. Ainsi, 2,8 millions de voix de plus (dont beaucoup sur ces deux listes) sont restées sans représentation parlementaire et ont probablement fait perdre bien des sièges à l'Ulivo. On peut raisonnablement soutenir que les électeurs de Di Pietro, aussi modérés et "non-de-gauche" soient-ils, sont potentiellement raccordables au centre-gauche, sans parler de la dimension "anti-berlusconienne" que cette liste a prise publiquement. Si l' Ulivo avait donc réussi à transiger ou à contracter un accord électoral avec Di Pietro, on peut penser qu'il aurait pu compter sur la quasi-intégralité du soutien électoral qui, finalement, est allé se perdre au profit de la liste Italia Dei Valori ${ }^{16}$.

Pour ce qui est de Démocrazia Europea, il est fort probable, au moins à la lumière des manœuvres pré-électorales de D'Antoni, qu'une éventuelle représentation parlementaire aurait joué en faveur de la Casa delle Libertà. Toutefois, si les éventuels sièges auraient pu être attribués au centre-droit, les votes obtenus concrètement par $\mathrm{DE}$ ont été en bonne part soustraits au centre-gauche (comme on l'a vu nettement dans les déplacements de voix au Sud et dans les îles, points de force du sicilien D'Antoni, qui y a obtenu respectivement 7 et $6,9 \%$ ) : en particulier, ces votes ont été soustraits aux forces centristes ex-démocrates-chrétiennes, qui avaient donné naissance, au sein de l'Ulivo, à la sub-coalition centriste de la Margherita, ou bien au PPI (dont D'Antoni fut un des plus prestigieux et puissants membres, du haut de sa fonction de secrétaire de la CISL, avant de fonder DE) ou à l'Udeur de Mastella.

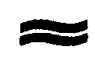

Les élections du 13 mai ont ouvert un cadre de stabilité institutionnelle potentielle et inédite. Le gouvernement semble voué à un long parcours. La gestion du pouvoir est certes le plus efficace ciment pour tenir ensemble une coalition comme la Casa delle Libertà, où les différences d'un parti à l'autre sont grandes et les intérêts très contrastés, et où la surpuissance de Berlusconi et de Forza Italia risque de créer des rancœurs chez les alliés. Mais les ressources que le président du Conseil peut distribuer sont suffisantes pour faire étouffer toute éventuelle protestation. Elles seront également en mesure, probablement, de désamorcer les mines représentées soit par le "conflit d'intérêt" (entre le Berlusconi l'entrepreneur et concessionnaire de réseaux télévisuels et le Berlusconi chef du gouvernement en mesure de favoriser quotidiennement les nombreuses sociétés dont il est propriétaire), soit par les poursuites judiciaires encore ouvertes à son encontre.

Sur le front des vaincus, l'incertitude règne. Rifondazione Comunista est renfermée au coin où elle s'est volontairement cachée, condamnée à une marginalité politique dont elle ne semble pas vouloir se départir. Les DS sont en plein chaos. D'Alema et le cercle politique d'origine communiste, quelles que soient les responsabilités qu'ils doivent assumer dans la réduction à un minimum de leur influence 


\section{HORS DOSSIER}

électorale, sont au seuil d'une dissolution organisationnelle. Ils ne semblent pourtant pas vouloir céder la place à de nouveaux dirigeants, moins bureaucratisés, qui existent certainement encore, même peu nombreux, au sein des DS. En novembre 2001, le Parti tient un congrès dont on ne peut pour l'instant attendre autre chose qu'un règlement de comptes entre les différentes tendances.
Les mouvements les plus intéressants sont à attendre de la Margherita, qui est en train de tenter de passer d'une coalition à un vrai parti unitaire, à l'appui de ses bons résultats électoraux. Mais ici aussi les résistances à la fusion, de la part de quelques membres de l'alliance, sont notoires et l'issue demeure assez incertaine. Pour l'instant (septembre 2001), nous avons perdu la trace d'Antonio di Pietro et de Sergio d'Antoni.

\section{Tableau 1 - Elections Keglalatives du 13 mal 2001 \\ Chambre des deputes - Contingent majoritaire. \\ Nombre de voix, \% des suffrages exprimés et nombre de sièges. \\ Comparaison avec les précédentes législatives.}

\begin{tabular}{|c|c|c|c|c|c|c|c|}
\hline \multirow[b]{2}{*}{$\begin{array}{l}\text { LISTES/ } \\
\text { COALITIONS }\end{array}$} & \multicolumn{2}{|c|}{13 mai 2001} & \multirow[b]{2}{*}{ SIEGES } & \multicolumn{4}{|c|}{21 avril 1996} \\
\hline & volx & $\%$ & & $\begin{array}{l}\text { LISTESI } \\
\text { COALITIONS }\end{array}$ & volx & $\%$ & SIEGES \\
\hline L'Ulivo & 16.289 .204 & 43,7 & 189 & $\begin{array}{l}\text { L'Ulivo + Rif. } \\
\text { Comunista }\end{array}$ & 16.908 .151 & 45,3 & 265 \\
\hline $\begin{array}{l}\text { Casa delle } \\
\text { Libertà }\end{array}$ & 16.918 .020 & 45,4 & 282 & $\begin{array}{l}\text { Polo per le } \\
\text { Libertà }\end{array}$ & 5.097 .220 & 40,5 & 169 \\
\hline $\begin{array}{l}\text { Lista Di } \\
\text { Pietro }\end{array}$ & 1.496 .110 & 4,0 & - & Lega Nord & 4.038 .511 & 10,8 & 39 \\
\hline $\begin{array}{l}\text { Democrazia } \\
\text { Europea }\end{array}$ & 1.313 .542 & 3,5 & - & $\begin{array}{l}\text { Fiamma } \\
\text { Tricolore }\end{array}$ & 629.522 & 1,7 & - \\
\hline $\begin{array}{l}\text { Pannella- } \\
\text { Bonino }\end{array}$ & 462.863 & 1,2 & - & Autres & 630.729 & 1,7 & 2 \\
\hline Autres & 804.351 & 2,2 & 4 & & & & \\
\hline $\begin{array}{l}\text { Suffrages } \\
\text { exprimés }\end{array}$ & 37.284 .090 & 100,0 & 475 & & 37.304 .133 & 100,0 & 475 \\
\hline Votes blancs & 1.514 .043 & 3,8 & & & 1.432 .888 & 3,5 & \\
\hline Votes nuls & 1.363 .366 & 3,4 & & & 1.740 .535 & 4,3 & \\
\hline Votants & 40.172 .536 & 81,4 & & & 40.496 .438 & 82,9 & \\
\hline Inscrits & 49.358 .947 & 100,0 & & & 48.846 .238 & 100,0 & \\
\hline
\end{tabular}


les élections législatives du 13 mai 2001 en Italie

\section{Tableau 2 - Electlons Lglalatives du 13 mal 2001}

Senat

Nombre de voix,\% des suffrages exprimés et nombre de sieges.

Comparaison avec les precédentes législatives.

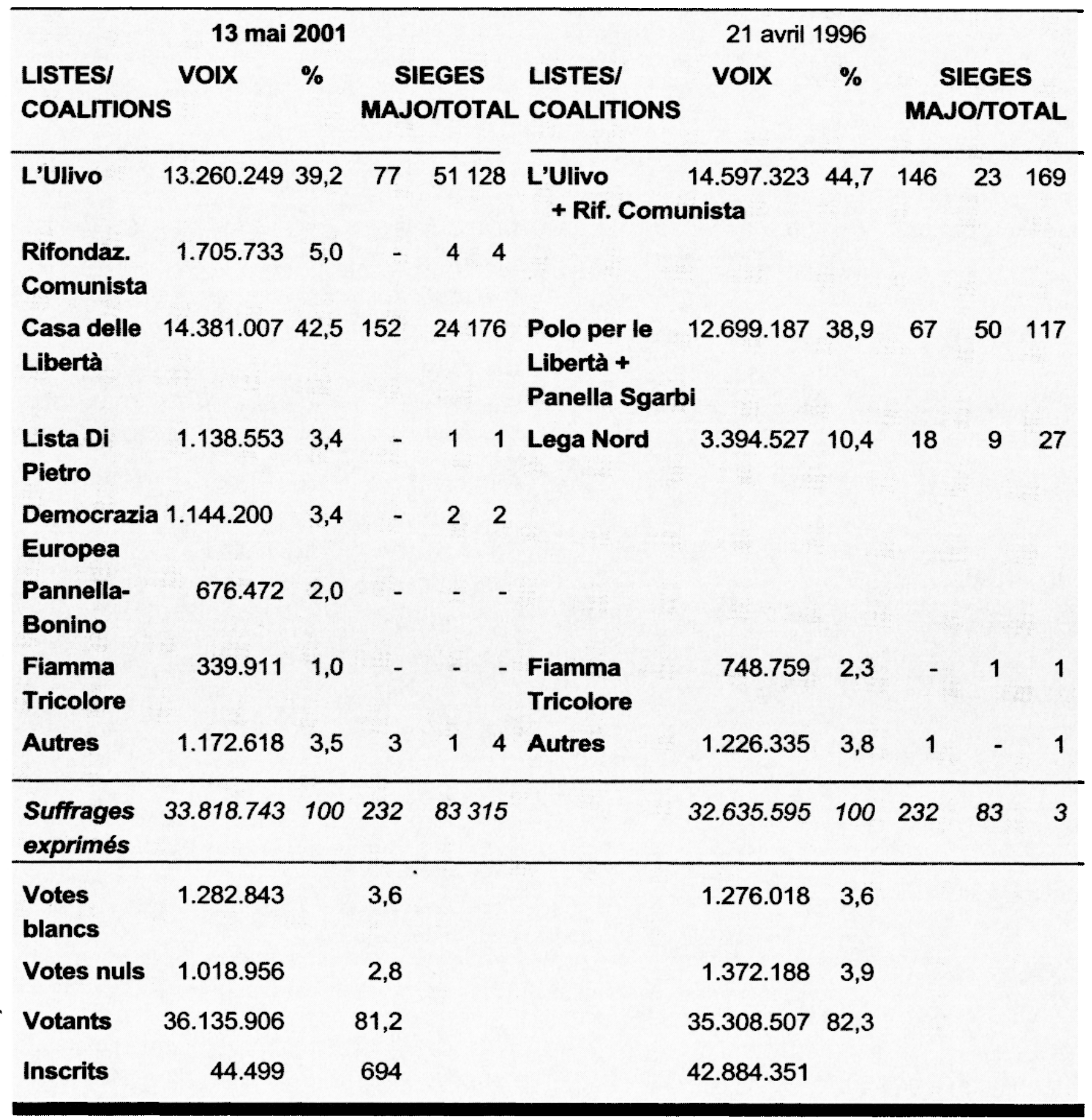


Tabloau 3 - Elections Ifglelatives du 13 mal 2001

Chambre dos difputes - Contingent proportionnel.

Nombre de voix, \% des suffrages exprimés et nombre de sieges.

Comparaison avoc les précédentes légistatives.

13 mai 2001

\begin{tabular}{|c|c|c|c|c|c|c|c|}
\hline $\begin{array}{l}\text { LISTESI } \\
\text { COALITIONS }\end{array}$ & volx & $\%$ & SIEGES & $\begin{array}{l}\text { LISTESI } \\
\text { COALITIONS }\end{array}$ & volx & $\%$ & GES \\
\hline $\begin{array}{l}\text { Democratici } \\
\text { di Sinistra (DS) }\end{array}$ & 6.147 .624 & 16,6 & 31 & PDS & 7.897 .044 & 21,1 & 26 \\
\hline \multirow[t]{2}{*}{ La Margherita } & 5.386 .950 & 14,5 & 27 & $\begin{array}{l}\text { POP-SVP-PRI- } \\
\text { UD-Prodi }\end{array}$ & 2.555 .082 & 6,8 & 4 \\
\hline & & & & Liste Dini & 1.627 .191 & 4,3 & 8 \\
\hline II Girasole & 804.488 & 2,2 & - & $\begin{array}{l}\text { Federazione } \\
\text { dei Verdi }\end{array}$ & 937.684 & 2,5 & - \\
\hline Comunisti Italiani & 619.912 & 1,7 & - & Autres & 37.974 & 0,1 & \\
\hline Total Ulivo & 12.958 .974 & 35,0 & 58 & Total Ulivo & 13.054 .975 & 34,8 & 38 \\
\hline \multirow[t]{2}{*}{ Comunista } & 1.868 .113 & 5,0 & 11 & $\begin{array}{l}\text { Rifondazione } \\
\text { Comunista }\end{array}$ & 3.215 .960 & 8,6 & 20 \\
\hline & & & & $\begin{array}{l}\text { Total Ulivo + } \\
\text { Rifond. Comuni }\end{array}$ & $\begin{array}{l}16.232 .961 \\
\text { sta }\end{array}$ & 43,3 & 58 \\
\hline Forza Italia & 10.923.146 & 29,5 & 62 & Forza Italia & 7.715 .342 & 20,6 & 37 \\
\hline \multicolumn{2}{|c|}{ Alleanza Nazionale4.459.397 } & 12,0 & 24 & Alleanza Nazion & ale5.875.391 & 15,7 & 28 \\
\hline Lega Nord & 1.461 .854 & 3,9 & - & & & & \\
\hline CCD-CDU & 1.193 .643 & 3,2 & - & CCD-CDU & 2.190 .019 & 5,8 & 12 \\
\hline Nuovo PSI & 352.853 & 1,0 & - & & & & \\
\hline \multirow[t]{3}{*}{ delle Libertà } & 18.390 .893 & 49,6 & 86 & $\begin{array}{l}\text { Total Polo per } \\
\text { le Libertà }\end{array}$ & 15.780 .752 & 42,1 & 77 \\
\hline & & & & Lega Nord & 3.777 .786 & 10,1 & 20 \\
\hline & & & & $\begin{array}{l}\text { Total Polo per } \\
\text { Libertà + Lega }\end{array}$ & 19.558 .538 & 52,2 & 97 \\
\hline
\end{tabular}

\begin{tabular}{lrrr}
\hline Liste Di Pietro & 1.443 .271 & 3,9 & - \\
Democrazia & 887.037 & 2,4 & -
\end{tabular}

Europea

\begin{tabular}{|c|c|c|c|c|c|c|c|}
\hline Pannella- Bonino & 831.199 & 2,2 & - & Pannella- Sgarbi & 701.033 & 1,9 & - \\
\hline Fiamma Tricolore & 142.894 & 0,4 & - & Fiamma Tricolore & 338.721 & 0,9 & - \\
\hline Autres & 578.443 & 1,5 & - & Autres & 663.712 & 1,7 & \\
\hline \multicolumn{2}{|c|}{ Suffrages exprimés 37.100 .824} & 100,0 & 155 & & 37.494 .965 & 100,0 & 155 \\
\hline Votes blancs & 1.688 .634 & 4,2 & & & 1.241 .498 & 3,1 & \\
\hline Votes nuls & 1.283 .772 & 3,2 & & & 1.659 .757 & 4,1 & \\
\hline Votants & 10.085.397 & 81,2 & & & 40.411 .692 & 82,9 & \\
\hline Inscrits & 9.362 .342 & & & & 48.744 .846 & & \\
\hline
\end{tabular}




\section{Notes}

1. La sortie de la Lega Nord de la majorité avait fait chuter après quelques mois le gouvernement de Berlusconi, qui avait remporté les élections de mars de la même année, ouvrant ainsi la voie au succès électoral du centregauche en 1996.

2. Comme on le sait, depuis 1993 une loi électorale "tendanciellement majoritaire" est en vigueur, sur la base de laquelle $75 \%$ des sièges de chacune des chambres sont attribués à l'issue d'une compétition entre candidats inscrits sur des listes uninominales, selon le principe "majoritaire sec" ("first past the post"). En particulier, pour l'élection des députés (630 au total), le territoire national est divisé en 475 collèges d'environ 100 à 150000 électeurs, qui accordent autant de sièges aux vainqueurs. Le reste ( $25 \%$ soit 155 sièges) est attribué aux listes de partis que dépassent nationalement le seuil de $4 \%$, avec une distribution nettement proportionnelle, qui favorise les partis qui ont obtenu le moins de sièges au quota majoritaire. Pour le Sénat ( 315 membres), la compétition majoritaire se joue sur 232 circonscriptions, alors que les 83 sièges restants sont attribués à la proportionnelle, sur une base régionale, aux candidats qui ont obtenu le plus de voix.

3. "Au Sénat, l' Ulivo + Rifondazione ont battu la Casa delle Libertà de $\mathbf{4 0 0 0 0 0}$ voix, mais le système électoral majoritaire a fait la différence. Ainsi, pour obtenir 3 sénateurs, Rifondazione a dû recueillir 1,7 millions de voix et, arithmétiquement, a entraîné la défaite de l'Ulivo dans 32 collèges" E. Berselli, R. Cartocci: "Il Bipolarismo realizzato", Il Mulino, $n^{\circ} 3,2001$, p. 452

4. Diamanti, I. "Vecchi e nuove subculture politiche", Il Mulino, $\mathrm{n}^{\circ}$ 4, 2001, p. 648

5. Floridia, A., "Il voto in Toscana: I'ultime regione rossa?", Congres Annuel de la Societte Italienne de Science Politique, Sienne, 13-15 septembre 2001

6. Ibid, p. 26. Un autre élément significatif que la recherche de Floridia met en évidence: alors qu'en 199618 communes toscanes votaient à plus de $50 \%$ pour le PDS et 70 entre 40 et $50 \%$, il n'y en a plus que 2 dans le premier cas et 46 dans le second en 2001.

7. Une première valorisation des résultats de cette recherche est sortie en septembre 2001 chez Il Mulino sous le titre "Perché ha vinto Berlusconi". Les informations que nous utilisons ici sont tirées de ITANES, "Radiografia di un voto", Il Mulino, $n^{\circ} 4,2001$, pp. 721-724.

8. Ibid., p. 722.

9. Ibid., p. 724.

10. Les données d'un sondage (ISCO/AC Nielsen-CRA) sur les seuls électeurs de Forza Italia confirment les révélations de l'enquête ITANES sur les points de force du centre-droit. En particulier, concernant le vote ouvrier, Forza Italia "n'apparaît pas seulement plus sous-représentée" par rapport au passé, mais le 13 mai a recueilli au sein de ces catégories "des pourcentages supérieurs à la moyenne $(+2 \%)$ ", R. Biorcio, "Forza Italia, parti de référence", Il Mulino, n 4, 2001 p. 625.

11. Gruppo ITANES, "Radiogafia di un voto", op. cit., p. 722.

12. Berlusconi s'est présenté à la télévision exhibant un "contrat" préparé par lui et demandant aux italiens d'y souscrire avec leur vote, où il devait s'employer à "tout mettre d'aplomb" en cinq ans, faute de ne pouvoir se représenter aux élections. La trouvaille du "contrat avec les italiens" (qui a fait rire beaucoup d'observateurs, sauf les électeurs du centre-droit, évidemment) reprenait le "contrat avec l'Amérique" lancé par le milliardaire Ross Perot dans sa candidature aux élections présidentielles américaines. Perot est, comme on le sait, le modèle politique dont s'inspira Berlusconi quand il descendit dans l'arène politique.

13. Sur la stratégie électorale de Berlusconi, voir les observations de G. Sani, op. cit.

14. Le combatif ancien procureur de Milan rendu célèbre par l'enquête sur la corruption politique dénommée "Mani Pulité" qui donna, au début des années 1990, le coup de grâce au vieux système des partis.

15. Pour la liste Di Pietro, l'exclusion a fait l'effet d'une gifle puisque le seuil de $4 \%$ n'a été manqué que de quelques voix (40000). Encore plus humiliante fut la décision du seul sénateur élu à la proportionnelle sur la liste Di Pietro de passer, quelques heures apress, dans les rangs de $1^{\text {" }}$ ennemi" : Berlusconi.

16. Rappelons que Di Pietro a également été Ministre et a été élu sénateur dans un collège rouge de Toscane; et qu'au sein de l'Ulivo, il avait contribué à la naissance d'un parti, I Democratici, dont il s'était éloigné à la suite de conflits de pouvoir peu reluisants... 